\title{
RELATIONSHIP BETWEEN THE AGE RANGE OF MAMMOGRAPHY AND THE BI-RADS CLASSIFICATION FOUND IN PATIENTS WITHOUT RISK FOR NEOPLASIA
}

Amanda Cristina de Souza Vieira1', Bianca Borges Martins¹, Matheus Nascimento Duarte1, Thiago de Moura Arruda ${ }^{1}$, Wglaison Paulo Araújo Sobral' ${ }^{1}$, Fabiana Cândida de Queiroz Santos Anjos' ${ }^{1}$ Priscila Ferreira Barbosa ${ }^{1}$

'Universidade de Gurupi - Gurupi (TO), Brazil.

Objective: The aim of this study was to analyze a relationship between the age range of mammography and BI-RADS, in patients without a high risk for neoplasia. Methodology: This is an epidemiological, cross-sectional, and retrospective study, based on the data from the Breast Cancer Information System, with the variables as follows: period, from January 2009 to July 2015; age group, from 30 to above 70 years old, distributed in groups of 10 years; BI-RADS category, from 0 to 6; high risk, not high risk. The collected data by using the chi-square test, with a significance level of 0.05 . Results: A total of 4,419,869 cases were observed. The age group that most performed the examination was 40-49 years old, representing $40.2 \%$ ( $n=1,777,924)$, followed by the age group $50-59$ years old, with $29.5 \%$ ( $n=1,305,317)$. The age group that did the least was above 70 years, with $5.9 \%(n=257,338)$. Among the BI-RADS classification, it can be noted that categories 1 and 2 were the most prevalent, with 45.8 and $40.3 \%$, respectively. There was a significant difference $(\mathrm{p}<0.05)$ in the incidence rates of all BI-RADS classification between the age groups: 30-39, 60-69, and above 70 years. The age groups of 40-49 and 50-59 years did not show a significant difference ( $p>0.005)$ in any of the BI-RADS classifications. Conclusion: It was observed in this research that there is no significant difference between the BI-RADS of mammograms performed in patients aged 50-59 and 40-49 years. In this context, the need for further investigation into the effectiveness of public health policy in effect is highlighted, where the likely increase in public costs with a greater number of mammograms does not equate to costly cancer treatment.

Keywords: Mammography; Clinical Protocols; Primary Prevention. 